\title{
Reformbedarf im schweizerischen Sexualstrafrecht
}

\author{
«Egoistisch, rücksichtslos, kaltherzig» - aber strafrechtlich nicht \\ relevant?
}

\section{Nora Scheidegger / Agota Lavoyer / Tamara Stalder *}

Anhand von fünf Fallbeispielen aus der kantonalen Praxis soll veranschaulicht werden, dass bei sexuellen Übergriffen nicht nur die den Sexualdelikten typischerweise inhärenten Beweisprobleme, sondern mitunter auch das materielle Recht schlicht keine Strafverfolgung ermöglicht. Diese Praxisbeispiele zeigen, dass sich Beschuldigte nach geltendem Recht bisweilen wissentlich über ein klares «Nein» hinwegsetzen können, ohne sich wegen Vergewaltigung oder sexueller Nötigung strafbar zu machen. Damit entkräftet die vorliegende Untersuchung die Meinung, dass das schweizerische Recht bereits heute alle Fälle nicht-einverständlicher sexueller Handlungen angemessen erfassen könne.

I. Einleitung .58

II. Der aktuelle rechtliche Schutz der sexuellen Selbstbestimmung...

1. Vorgaben gemäss EMRK und Istanbul-Konvention ..........................................59

2. Problematik der Bestimmungen des Schweizerischen Strafgesetzbuches.......60

III. Veranschaulichung der Problematik anhand von weiteren kantonalen Praxisbeispielen

1. Methodik 62

2. Kein Widerstand, kein Nötigungsmittel, keine Vergewaltigung. 63

3. Kein Widerstand, kein Vorsatz, kein Sexualdelikt?..........................................68

IV. Kritische Diskussion und kriminalpolitische Einbettung.....................................70

1. Materielles Recht als Hürde ...........................................................................70

2. Reformvorschläge................................................................................... 72

3. Falsche Versprechungen?........................................................................... 73

Zitiervorschlag: Nora Scheidegger/Agota Lavoyer/Tamara Stalder, Reformbedarf im schweizerischen Sexualstrafrecht, in: sui-generis 2020, S. 57

URL: $\quad$ sui-generis.ch/122

DOI: $\quad$ https://doi.org/10.21257/sg.122

\footnotetext{
* Nora Scheidegger, Senior Researcher MPI Freiburg im Br./ Agota Lavoyer, Stv. Leiterin und Beraterin bei Lantana, Fachstelle Opferhilfe bei sexueller Gewalt - Stiftung gegen Gewalt an Frauen und Kindern in Bern / Tamara Stalder, Hilfsassistentin Universität Bern. Wir bedanken uns bei den Betroffenen, die sich dazu bereit erklärt haben, dass ihr Fall für diesen Aufsatz verwendet werden darf, bei den RechtsanwältInnen und Opferhilfeberaterinnen für die Zusendung der Fälle sowie bei Prof. Jonas Weber, Prof. Christopher Geth, Prof. Martino Mona, Dr. Jann Schaub und Nicolas Leu für die wertvollen Hinweise und Anregungen.
}

Dieses Werk ist lizenziert unter einer Creative Commons Namensnennung - Weitergabe unter gleichen Bedingungen 4.0 International Lizenz. 


\section{Einleitung}

1 «Sexualität muss einvernehmlich sein!» Diese Aussage ist nicht nur Grundlage von Sensibilisierungskampagnen und dem Aufklärungsunterricht in Schulen, sondern umschreibt auch eine menschenrechtliche Vorgabe. Die Schweiz ist aufgrund der Rechtsprechung des EGMR ${ }^{1}$ und der sog. Istanbul-Konvention ${ }^{2}$ verpflichtet, alle nicht-einverständlichen Sexualkontakte umfassend und angemessen unter Strafe zu stellen sowie bei entsprechenden Übergriffen eine effektive Strafverfolgung zu gewährleisten. Insbesondere mit Blick auf die IstanbulKonvention wurden nun in jüngerer Zeit verschiedene Massnahmen im Bereich der Strafverfolgung von sexuellen Übergriffen gefordert, unter anderem auch eine Reform des materiellen Sexualstrafrechts. ${ }^{3}$ Begründet wurde dies damit, dass das geltende Sexualstrafrecht dazu beitrage, dass Fälle von nichteinverständlichen sexuellen Handlungen unbestraft bleiben. Diese Frage, d.h. ob und inwieweit im schweizerischen Sexualstrafrecht Reformbedarf besteht, ist derzeit Gegenstand öffentlicher Diskussionen 4 und politischer Vorstösse. 5

Urteil des EGMR 39272/98 vom 4. Dezember 2003 (M.C. gegen Bulgarien), § 166.

2 Übereinkommen des Europarats zur Verhütung und Bekämpfung von Gewalt gegen Frauen und häuslicher Gewalt (Istanbul-Konvention), abgeschlossen in Istanbul am 11. Mai 2011 (SR 0.311.35).

3 Vgl. Nora Scheidegger, Das Sexualstrafrecht der Schweiz - Grundlagen und Reformbedarf, Diss. Bern 2018, Rn. 587 ff.; Petition von Amnesty International; Gastbeitrag von 22 StrafrechtsprofessorInnen, Übergriffe angemessen bestrafen - Das Schweizer Sexualstrafrecht muss revidiert werden, in: TagesAnzeiger vom 4. Juni 2019; Medienmitteilung «Nationales Fachgremium Sexuelle Gewalt an Frauen» vom 5. September 2019.

4 Einen Reformbedarf bestreitend insbes. Prof. Marcel A. Niggli in Katharina Fontana, Sex nach
2 Ziel des vorliegenden Beitrags ist es daher zu untersuchen, ob und inwiefern neben den typischen Beweisschwierigkeiten auch das materielle Strafrecht als Ursache für die Straflosigkeit von sexuellen Übergriffen - hier verstanden als nichteinverständliche sexuelle Handlungen an einer Person - thematisiert werden muss. Dafür sollen zunächst die völkerrechtlichen Bestrafungspflichten hinsichtlich sexueller Gewalt kurz dargestellt und anschliessend die Diskrepanz zwischen dem Umfang der durch die Istanbul-Konvention zu bestrafenden Verhaltensweisen und dem, was nach schweizerischem Strafrecht verfolgt werden kann, thematisiert werden (II.). Anschliessend soll die so festgestellte Lücke anhand einer Auswahl von Fällen aus der kantonalen Praxis veranschaulicht werden. Besonders deutlich kann diese Diskrepanz und ihre praktischen Konsequenzen anhand von Endentscheiden aufgezeigt werden, bei denen zwar erstellt war, dass der Beschuldigte eine nichteinverständliche sexuelle Handlung am Opfer vornahm, eine Verurteilung aber daran scheiterte, dass das Verhalten nicht unter einen der Tatbestände des geltenden Sexualstrafrechts subsumiert werden konnte (III.). Eine solche Untersuchung erscheint auch deshalb notwendig, weil diese Endentscheide oftmals nicht in öffentlich zugänglichen Datenbanken abrufbar sind und damit für den

Vertrag, in: Weltwoche vom 28. Mai 2019; Gastbeitrag von 32 StrafverteidigerInnen, Professorale Fake News zum Sexualstrafrecht, in: TagesAnzeiger vom 22. Juni 2019; Interview mit RA Tanja Knodel im Beitrag von Brigitte Hürlimann, Wollen die Anwälte, dass Vergewaltiger ohne Strafe bleiben? in: Republik vom 12. Juli 2019; Laura Jetzer/Diego R. Gfeller, Gegen eine rechtsstaatlich problematische Verschärfung des Sexualstrafrechts, in: NZZ vom 31. Januar 2020.

5 Vgl. etwa die Interpellationen 18.3889 und 19.3587. 
öffentlichen Diskurs weitgehend unsichtbar bleiben. Zuletzt sollen die gewonnenen Erkenntnisse kritisch diskutiert und in die aktuelle kriminalpolitische Debatte eingebettet werden (IV.). Dabei soll dargelegt werden, dass die geltende Rechtslage nach Erfahrung von OpferhilfeBeratungsstellen auch dazu führt, dass Opfer von vornherein auf eine Anzeige verzichten und so möglicherweise selbst Vorfälle im Dunkelfeld verbleiben, die bereits heute justiziabel wären.

\section{Der aktuelle rechtliche Schutz der sexuellen Selbstbestimmung}

\section{Vorgaben gemäss EMRK und Istanbul-Konvention}

Recht auf sexuelle Selbstbestimmung ist ein grund- und menschenrechtlich geschütztes Recht und hat eine positive und eine negative Dimension - die Freiheit zu gewollter Sexualität sowie die Freiheit vor ungewollter Sexualität, vor sexueller Gewalt und sexuellem Missbrauch. ${ }^{6}$ Letzteres kann als Abwehrrecht verstanden werden, «im Sinne eines 〈Vor-Angriffen-bewahrt-bleibens〉, eines 〈Sich-verweigern-könnens〉, als Freiheit vor Fremdbestimmung auf sexuellem Gebiet.»7 Eine Freiheit zu Sexualität kann es nur soweit geben, als nicht die Freiheiten anderer Personen betroffen sind. Die Einwilligung wiederum ist Ausfluss des Selbstbestimmungsrechts und mit diesem untrennbar verknüpft: Indem wir in eine sexuelle Handlung einwilli-

Eingehend dazu Scheidegger (Fn. 3), Rn. $185 \mathrm{ff}$. unter Hinweis auf Urteil des EGMR 8978/80 vom 26. März 1985 (X. und Y. gegen die Niederlande), § 23.

7 So bereits Brigitte Sick, Sexuelles Selbstbestimmungsrecht und Vergewaltigungsbegriff, Diss. Berlin 1993, S. 86; eingehend Scheidegger (Fn. 3), Rz. $26 \mathrm{ff}$. gen, verwirklichen wir unser Selbstbestimmungsrecht. Umgekehrt ist das Selbstbestimmungsrecht verletzt, wenn keine Einwilligung vorliegt. ${ }^{8}$ Ohne Einwilligung des anderen handelt es sich bei einem Sexualakt nicht um ein legitimes Entfalten der eigenen Sexualität, sondern um Usurpation. 9

4 In seinem wegweisenden Entscheid M.C. gegen Bulgarien hat denn auch der EGMR festgehalten, dass die Mitgliedstaaten die positive Pflicht haben, alle nicht-einverständlichen Sexualakte zu verfolgen und zu bestrafen, und zwar auch dann, wenn das Opfer sich nicht körperlich zur Wehr gesetzt hat.10 Auch aus der 2017 von der Schweiz ratifizierten Istanbul-Konvention ergibt sich die Verpflichtung, jeden Sexualkontakt unter Strafe zu stellen, der nicht von einem freiwillig erteilten Einverständnis getragen wird. ${ }^{11}$ Damit wird den Staaten die Einführung des Konsensprinzips (im Sinne einer «Nein-heisst-Nein»- oder «Nur-Ja-heisst-Ja»-Regel) im Sexualstrafrecht nahegelegt, wie auch die bisher ergangenen Empfehlungen des zuständi-

8 Alan Wertheimer, Consent to Sexual Relations, Cambridge 2003, S. 31; Stuart Green, Lies, Rape, and Statutory Rape, in: A. Sarat (Hrsg.), Law and Lies: Deception and Truth-Telling in the American Legal System, Cambridge 2015, S. 211: «One of the principal indicators for determining whether an individual's sexual autonomy is respected is consent.»; eingehend auch Scheidegger (Fn. 3) Rz. 40 ff. m.w.N.

9 Joachim Renzikowski, Lücken beim Schutz der sexuellen Selbstbestimmung aus menschenrechtlicher Sicht, Stellungnahme in der Anhörung zu dem Antrag «Artikel 36 der Istanbul-Konvention umsetzen - bestehende Strafbarkeitslücken bei sexueller Gewalt und Vergewaltigung schließen», BT-Drs. 18/1969, S. 4.

10 Urteil des EGMR 39272/98 vom 4. Dezember 2003 (M.C. gegen Bulgarien), § 166.

11 Vgl. Art. 36 Istanbul-Konvention (Fn. 2). 
gen Überwachungsgremiums zeigen.12 Zahlreiche europäische Länder haben jedenfalls aufgrund der IstanbulKonvention ihre sexualstrafrechtlichen Bestimmungen überarbeitet und ergänzt. ${ }^{13}$ So wurde etwa in Schweden, Island, Deutschland und Österreich das Sexualstrafrecht kürzlich reformiert, 14 in anderen Ländern wurden bereits entsprechende Absichtserklärungen abgegeben.15 Der schweizerische Bundesrat hingegen stellte sich bislang auf den Standpunkt, dass das geltende Recht in der Schweiz den Anforderungen bereits genügt - eine Einschätzung, der zahlreiche StrafrechtsprofessorInnen widersprochen haben. ${ }^{16}$

\section{Problematik der Bestimmungen des Schweizerischen Strafgesetzbuches}

5 Während in anderen europäischen Ländern Vergewaltigung zunehmend als «Sex ohne Einwilligung» definiert wird, kennt das Schweizerische Strafgesetzbuch keinen als Verbrechen oder Verge-

12 Grevio Baseline Evaluation Report Denmark, 2017, Rn. 177: "GREVIO strongly encourages the Danish authorities to move away from the current sexual violence legislation and base it on the notion of freely given consent as required by Article 36, paragraph 1 of the Istanbul Convention.» ̈̈hnlich auch Grevio Baseline Evaluation Report Finland, 2019, Rn. 165.

13 Vgl. die Übersicht von Amnesty International Schweiz, Reformen in europäischen Ländern verdeutlichen Handlungsbedarf, vom 10. September 2019.

14 Vgl. Kap. 6 § 1 SWE-StGB; Art. 194 ISL-StGB; $\S 177$ D-StGB; § 205a A-StGB.

15 Vgl. etwa die Mitteilung der holländischen Regierung vom 2. Mai 2019; Stephen Burgen, Spain to introduce 'yes means yes' sexual consent law, in: The Guardian vom 18. Juli 2018; Mitteilung Amnesty International betreffend Dänemark: Government commitment to amend rape law is a hard-won victory.

16 Vgl. BBl 2017 241, wonach Artikel 36 der Istanbul-Konvention im schweizerischen Strafrecht abgedeckt werde, kritisch dazu Gastbeitrag StrafrechtsprofessorInnen (Fn. 3). hen ausgestalteten Tatbestand, der nichteinverständliche Sexualkontakte per se unter Strafe stellt. Insbesondere Art. 189 und 190 StGB (Vergewaltigung und sexuelle Nötigung) sind als Nötigungsdelikte ausgestaltet, es ist also zusätzlich zur fehlenden Einwilligung erforderlich, dass die Täterschaft das Opfer nötigt. ${ }^{17}$ Das hat zur Konsequenz, dass nach dem geltenden materiellen Strafrecht (bei sexuell mündigen Menschen) eine sexuelle Handlung ohne Einwilligung nur dann als erhebliches Unrecht anerkannt wird, wenn $\mathrm{zu}$ der fehlenden Einwilligung weitere Umstände hinzutreten, nämlich die Anwendung eines Nötigungsmittels (Art. 189 und 190 StGB), die Ausnutzung der Urteils- oder Widerstandsunfähigkeit (Art. 191 StGB) oder die Ausnutzung eines Abhängigkeitsverhältnisses oder einer Notlage (Art. 188, 192 und 193 StGB). Konstellationen, in denen diese zusätzlichen Umstände nicht vorliegen, können - ein rechtzeitig gestellter Strafantrag vorausgesetzt - höchstens als sexuelle Belästigung (Art. 198 StGB) bestraft werden. ${ }^{18}$ Dies widerspricht der Vorgabe der Istanbul-Konvention, wonach nichteinverständliche sexuelle Handlungen von Amtes wegen verfolgt und angemessen bestraft werden müssen. ${ }^{19}$

6 Dass das Fehlen eines (Vergewaltigungs-) Tatbestandes, der das schlichte Handeln gegen bzw. ohne den Willen des Opfers angemessen unter Strafe stellt, zu unbefriedigenden Ergebnissen führt, zeigen jene Fälle, die man als «sexuelle Übergriffe ohne Nötigung» bezeichnen könnte. Gemeint sind Konstellationen, in de-

17 Eingehend Scheidegger (Fn. 3), Rz. 592 ff.

18 Kritisch Scheidegger (Fn. 3), Rz. 598 ff.

19 Vgl. Art. 41 sowie Art. 55 Istanbul-Konvention (Fn. 2); Scheidegger (Fn. 3), Rz. 599 ff. 
nen der Täter am passiv bleibenden bzw. ihn bloss verbal abweisenden Opfer sexuelle Handlungen vornimmt, dieses aber mangels Widerstands nicht zu nötigen braucht. Dem bundesgerichtlichen Entscheid 6B_912/2009 vom 22. Februar 2010 lag ein solches Geschehen zugrunde: Das Opfer «wehrte» sich lediglich verbal gegen den ungewollten Geschlechtsverkehr, zu weiteren Abwehrhandlungen fühlte sie sich nicht in der Lage - sie sei zu schockiert und verblüfft gewesen, wie sie selber angab. ${ }^{20}$ Der Beschuldigte musste also weder Gewalt anwenden noch sie mittels Drohung zur Duldung des Geschlechtsverkehrs bewegen. Auch das dritte bei Art. 189 und 190 StGB statuierte Nötigungsmittel, das sog. «Unter-Psychischen-Druck-Setzen», lag nach Auffassung des Kantons- und Bundesgerichts nicht vor. Es wäre dem Opfer nach Ansicht der Gerichte durchaus weitergehender Widerstand zumutbar gewesen, etwa durch physische Gegenwehr oder durch Verlassen des Zimmers. Damit fehlte es am Tatbestandsmerkmal des Nötigungsmittels und es musste ein Freispruch vom Vorwurf der Vergewaltigung ergehen. Das Bundesgericht hielt in seinem Entscheid ausdrücklich fest:

«Der blosse Vollzug des Geschlechtsverkehrs gegen den vorgängig geäusserten Willen [des Opfers] bzw. eine nur geringfügige Kraftaufwendung genügt aufgrund des unbeeinträchtigten physischen und psychischen Zustands [des Opfers] demzu-

20 Vgl. dazu die Ausführungen im vorinstanzlichen Urteil des Kantonsgerichts Wallis P1 0866 vom 17. September 2009, E. 6.e.cc.bbb.: «Ihre Passivität erklärte sie damit, dass sie über sein Verhalten schockiert gewesen sei (...) bzw. 〈baff〉 (...). Sie sei sich wie eine Puppe vorgekommen (...).» folge nicht für den Tatbestand der Vergewaltigung.»21

7 Dass der Beschuldigte das sexuelle Selbstbestimmungsrecht der Frau (zumindest in zivilrechtlicher Hinsicht) vorsätzlich und erheblich verletzt hatte, war dabei unbestritten. ${ }^{22}$ Der Frau wurde deshalb eine Genugtuungssumme von CHF 3000.00 zugesprochen, was wie folgt begründet wurde:

«Vorliegend hat der [Beschuldigte] das Persönlichkeitsrecht [des Opfers] auf sexuelle Freiheit und Selbstbestimmung verletzt, indem er, wie festgestellt, in der fraglichen Nacht den wiederholten $\mathrm{Ge}$ schlechtsverkehr gegen den für ihn erkennbaren Willen [des Opfers] vollzog. Dies stellt einen widerrechtlichen, schweren Eingriff in deren Persönlichkeit dar, wobei das Verschulden des [Beschuldigten] ohne weiteres zu bejahen ist.»23

8 Dieser Entscheid vermittelt einen ersten Eindruck davon, dass es Fälle von nichteinverständlichen Sexualkontakten gibt, die mit dem Instrumentarium des geltenden Rechts nicht angemessen erfasst werden können. Nachfolgend soll anhand weiterer Fallbeispiele aus der Praxis gezeigt werden, dass es sich bei die-

21 Urteil des Bundesgerichts 6B_912/2009 vom 22. Februar 2010 E. 2.1.4.; vgl. auch Urteil des Bundesgerichts 6B_1078/2009 vom 13. Dezember 2010 E. 3.4.: «Zur Erfüllung der beschriebenen Tatbestände [Art. 189 und 190 StGB] reicht ein fehlendes Einverständnis (...) nicht aus.»

22 Insofern ist der von Jetzer/Gfeller (Fn. 4) geäusserte Vorwurf, der Fall sei überhaupt nicht geeignet, die problematische Rechtslage aufzuzeigen, nicht berechtigt.

23 Urteil des Kantonsgerichts Wallis P1 o8 66 vom 17. September 2009, E. 7.b. 
sem Urteil nicht um einen Einzelfall handelt.

\section{Veranschaulichung der Problematik anhand von weiteren kantonalen Praxisbeispielen}

\section{Methodik}

\section{a) Vorgehensweise der Erhebung}

9 Ende 2019 wurden neun OpferhilfeBeratungsstellen und ein kleinerer Kreis von RechtsanwältInnen angeschrieben und um die Zusendung von vollständig anonymisierten Endentscheiden gebeten, bei denen die materielle Rechtslage die (mit-)entscheidende Hürde für die strafrechtliche Verfolgung des Beschuldigten darstellte. Um eine Retraumatisierung der Opfer - etwa durch Wiedererkennen des «eigenen» Falls in der Publikation zu verhindern, wurden die AnwältInnen gebeten, vor der Übermittlung der Unterlagen die Zustimmung ihrer Klientinnen einzuholen. ${ }^{24}$ Zur Verfügung gestellt wurden innert kurzer Frist 28 anonymisierte Kopien von rechtskräftigen Endentscheiden aus mehreren Kantonen. Bei fehlender Begründung der Entscheide wurden Fallbeschreibungen der Anwältinnen eingeholt. Zusätzlich wurden auch Fälle verwendet, die bereits in öffentlich zugänglichen kantonalen Entscheidsammlungen publiziert worden

Einige der Angefragten berichteten zwar von geeigneten Fällen, verzichteten aber auf die Übermittlung, weil sie die Klientinnen nicht erreichen konnten oder es nicht für vertretbar hielten, sie erneut mit dem Fall zu konfrontieren. Andere Opfer wurden zwar angefragt, baten aber darum, dass «ihr» Fall nicht für die Publikation verwendet wird. Diese Entscheidungen wurden selbstverständlich respektiert. sind. ${ }^{25}$ Abgesehen von letzteren werden die uns zugesandten Verfügungen und Urteile vorliegend derart wiedergegeben, dass keinerlei Rückschlüsse auf den jeweiligen Kanton möglich sind, damit weder einzelne kantonale Staatsanwaltschaften oder Gerichte hervorgehoben werden noch die Identifizierung der Opfer ermöglicht wird. Aus Platzgründen wurden aus dieser Fallsammlung nur jene Entscheide für den Beitrag ausgewählt, anhand derer sich die problematische Rechtslage besonders gut veranschaulichen lässt. ${ }^{26}$

\section{b) Einschränkungen dieser Vorgehensweise}

10 Die vorliegende Untersuchung ist aus offenkundigen Gründen nicht geeignet, ein repräsentatives Abbild der Verfahrenswirklichkeit bei Sexualdelikten zu zeichnen. Aufgrund der gewählten Vorgehensweise lässt sich nicht bestimmen, in wie vielen Fällen prozentual die Strafverfolgung an der hier als problematisch identifizierten Rechtslage scheitert. Anzumerken ist hierbei, dass nach Erfahrung von Opferhilfe-Beratungsstellen solche Fälle mitunter gar nicht erst angezeigt werden, nachdem die Betroffenen über die Rechtslage informiert worden sind.

Dafür wurden die online zugänglichen Sammlungen der zweitinstanzlichen Gerichte der Kantone Zürich, Basel-Landschaft, Basel-Stadt, St. Gallen und Bern nach den Stichworten "Art. 190 StGB» und «Art. 189 StGB» durchsucht, wobei der Zeitraum 2010 bis 2020 berücksichtigt wurde.

26 Nicht verwendet wurden insbesondere die Fälle, in denen die Vorgehensweise des Beschuldigten u.E. durchaus als «Nötigung» i.S.v. Art. 189 und 190 StGB hätte qualifiziert werden können. 
11 Bei einigen der uns zugesandten Entscheide war der Sachverhalt noch nicht hinreichend erstellt. Dennoch können auch solche Verfügungen Aufschluss über die problematische Rechtslage geben, wenn die verfügende Staatsanwaltschaft als Einstellungsgrund angibt, dass selbst wenn man vollumfänglich auf die Aussagen des Opfers abstellen würde, eine Strafbarkeit des Beschuldigten ohnehin nicht gegeben wäre - womit die Unzulänglichkeit des materiellen Rechts unabhängig von der Beweisproblematik sichtbar wird.

\section{Kein Widerstand, kein Nötigungs- mittel, keine Vergewaltigung}

\section{a) Ohne Nötigungsmittel keine Vergewaltigung bzw. keine sexuelle Nötigung}

Im öffentlichen Diskurs rund um die Notwendigkeit einer Reform des Sexualstrafrechts wird häufig auf zwei Bundesgerichtsurteile verwiesen. ${ }^{27}$ In beiden Fällen handelte der Beschuldigte offenkundig gegen den Willen der betroffenen Frau, setzte jedoch (da mangels Widerstand der Frau auch nicht notwendig) kein Nötigungsmittel ein. Damit fehlte es aber an einem objektiven Tatbestandsmerkmal von Art. 190 StGB, weshalb jeweils ein Freispruch ergehen musste. Anhand der uns übermittelten Fälle wird ersichtlich, dass diese höchstrichterliche Rechtsprechung nicht «veraltet» ist oder Ausnahmecharakter aufweist, ${ }^{28}$ sondern

27 Urteile des Bundesgerichts 6B_912/2009 vom 22. Februar 2010 und 6B_311/2011 vom 19. Juli 2011.

28 Vgl. Aussage der interviewten Anwältin im Diskussionsbereich zum Interview in der «Republik» (Fn. 4):«[E]s ist überaus gefährlich, aufgrund eines Bundesgerichtsentscheides, der bald 10 - jährig ist, sie die Praxis der unteren Instanzen sowie der Staatsanwaltschaften bestimmt. Diese Urteile stehen auch im Einklang mit der sonstigen Rechtsprechung des Bundesgerichts, wonach der Beschuldigte den dem Opfer «zumutbaren» Widerstand brechen müsse, damit sein Verhalten unter Art. 189 und 190 StGB subsumiert werden kann. ${ }^{29}$

13 Zur Veranschaulichung nachfolgend ein Fall, bei dem die Beweisproblematik keine entscheidende Rolle gespielt hat, sondern eine Einstellung deshalb erging, weil das Verhalten des Beschuldigten nicht unter die aktuellen Sexualstraftatbestände subsumiert werden konnte.

\section{aa) Fall Nr. 1: Sachverhalt}

14 Das 19-jährige Opfer wurde gemäss ihren Angaben vom Lebensgefährten ihrer Mutter, den sie «Papi» nannte und der seit dem 4. Lebensjahr für sie sorgte, geweckt. Er habe sich zu ihr ins Bett gelegt und plötzlich angefangen, sie an den Brüsten zu berühren. Dann habe er ihr zwischen die Beine gegriffen, sie im Intimbereich zu streicheln begonnen. Er habe sie schliesslich auf den Rücken gedreht, sie entkleidet und im Intimbereich geleckt. Sie habe sich - aus Angst - nicht gewehrt, sondern schlafend gestellt und sich zwei, drei Mal auf die Seite gedreht, um ihm zu vermitteln, dass sie die sexu-

ein Gesetz und strafrechtliche Prinzipien ändern zu wollen.»

29 Vgl. BGE 131 IV 167 E. 3.1 S. 170 f. mit Hinweisen; eingehend zum Ganzen auch Urteil des Bundesgerichts 6B_302/2017 vom 25. Oktober 2017 E. 1.2.1: «Bei allen Nötigungsmitteln ist eine erhebliche Einwirkung auf die Freiheit der sexuellen Selbstbestimmung erforderlich. (...) [Es ist] zu berücksichtigen, dass Erwachsenen mit entsprechenden individuellen Fähigkeiten in der Regel eine stärkere Gegenwehr zuzumuten ist als Kindern.» 
ellen Handlungen nicht wollte. Der Beschuldigte war insofern geständig, als er die sexuellen Handlungen (mehrheitlich) zugab, er behauptete jedoch, das Opfer sei einverstanden und während des Vorgangs erregt gewesen.

\section{bb)Ausgang des Verfahrens}

Obwohl auch die Staatsanwaltschaft davon ausging, dass trotz fehlender Nötigungshandlung «nicht davon die Rede sein» könne, dass das Opfer eingewilligt habe, sondern vielmehr durch die Handlungen des Beschuldigten in ihrer sexuellen und psychischen Integrität (zumindest zivilrechtlich) «schwer verletzt» worden sei, wurde das Verfahren gegen den Beschuldigten eingestellt. Dessen Verhalten sei zwar «verwerflich» und «persönlichkeitsverletzend» gewesen, aber strafrechtlich nicht relevant.

Eine sexuelle Nötigung nach Art. 189 StGB liege nicht vor, weil kein Nötigungsmittel eingesetzt worden sei. Der Beschuldigte habe die junge Frau weder bedroht noch Gewalt angewendet und sie auch nicht zum Widerstand unfähig gemacht. Auch das Vorliegen einer besonderen Drucksituation (i.S. des Nötigungsmittels «Unter-psychischen-DruckSetzen») sei nicht ersichtlich. Gemäss Aussagen der Beteiligten habe die Geschädigte «es» vielmehr einfach über sich ergehen lassen, so dass der Beschuldigte keinen Widerstand zu überwinden hatte. Auch eine Strafbarkeit wegen eines anderen Sexualdelikts kam nicht in Betracht: Da die junge Frau nicht tatsächlich geschlafen hatte und auch sonst nicht widerstandsunfähig im Sinne von Art. 191 StGB war, liege keine Schändung vor. Auch stelle das Verhalten keine versuchte Schändung dar, da der Beschul- digte nach eigenen Angaben davon ausging, dass die junge Frau während des Geschehens wach war. Verworfen wurde auch der Tatbestand des Art. 193 StGB, da es am dafür erforderlichen ausgeprägten Machtgefälle zwischen Opfer und Beschuldigtem fehlte.

17 Eine mögliche Strafbarkeit nach Art. 198 StGB wurde nicht thematisiert. Aufgrund der Angaben zum zeitlichen Ablauf der Geschehnisse dürfte es aber am rechtzeitig gestellten Strafantrag gefehlt haben.

\section{b) Nein heisst Nein?}

18 In der aktuellen Debatte um eine Reform des Sexualstrafrechts wird behauptet, dass «Nein» bereits heute «Nein» heisse, mithin das Übergehen einer kommunizierten Ablehnung bereits heute von Gerichten als Vergewaltigung gewertet würde.30 Die uns zugesandten Fälle zeigen, dass dem nicht so ist: Die Staatsanwaltschaften und Gerichte kamen - in Anlehnung an die bundesgerichtliche Rechtsprechung - jeweils zum Schluss, dass das blosse Übergehen eines «Neins» oder das blosse Handeln ohne Einwilligung gerade nicht für Art. 189 bzw. 190 StGB ausreicht: Dies wurde in einem zweitinstanzlichen Urteil prägnant festgehalten:

«Zu ergänzen ist diesbezüglich allerdings, dass auch Sex, der gegen den Willen der Betroffenen erfolgt, noch nicht zwingend eine strafbare Handlung darstellt. Sowohl beim Tatbestand der sexuellen Nötigung

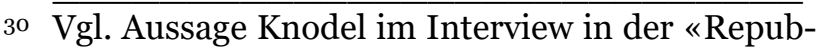
lik» (Fn. 4): «Das gilt heute schon, nach dem geltenden Sexualstrafrecht. Ein Nein ist ein Nein. Wenn jemand Nein sagt zur sexuellen Handlung, darf sich der andere nicht darüber hinwegsetzen. Die vorgeschlagene Änderung ist unnötig (...).» 
als auch bei demjenigen der Vergewaltigung sind dafür auf der objektiven Tatbestandsseite ein Tat-mittel wie Gewalt, Drohung, Unterpsychischen-Druck-Setzen oder ZumWiderstand-unfähig-Machen sowie das Tatbestandsmerkmal der Kausalität zwischen Tatmittel und Taterfolg Voraussetzung. (...) [D]ie blosse Missachtung des Willens [vermag] keinesfalls einen von der Praxis geforderten massgeblichen psychischen Druck zu erzeugen.»31

Dieses Zitat widerspiegelt und präzisiert die oben erwähnte Rechtsprechung, wonach ein entgegenstehender Wille, bzw. ein Widerstand des Opfers, gebrochen werden müsse. Zwar werden an diesen entgegenstehenden Willen keine sehr hohen Anforderungen gestellt - so kann es etwa bereits genügen, dass das Opfer die Beine fest zusammenpresst, so dass der Beschuldigte diese auseinanderdrücken muss. ${ }^{2}$ Trotz dieser relativ «opferfreundlichen» Auslegung bleibt es aber dabei, dass vom Opfer der ihm vermeintlich «zumutbare» Widerstand gefordert wird. Fehlt dieser, ist regelmässig kein Nötigungsmittel erforderlich und der Tatbestand der Vergewaltigung folglich nicht erfüllt. Die beiden folgenden Fallbeispiele zeigen dies gut auf: 4601490 vom 16. September 2014, S. 12 und 14.

32 Vgl. etwa Urteil des Bundesgerichts 6B_993/2013 vom 17. Juli 2014 E. 3.4.

\section{aa) Fall Nr. 233: Sachverhalt}

20 Der Beschuldigte versuchte während etwa 20 Minuten, seine Bekannte nach zunächst einvernehmlichem «Rummachen» zum Geschlechtsverkehr zu überreden und mit seinem Penis in sie einzudringen. Dabei teilte sie ihm wiederholt mit, dass sie keinen Geschlechtsverkehr haben möchte. Durch ihre abweisende Körperhaltung gelang ihm das Eindringen zunächst nicht. Daraufhin behauptete er ihr gegenüber, er habe sie nun entjungfert. Diese - falsche - Mitteilung löste beim Opfer dann offenbar insofern eine Reaktion aus, dass der Beschuldigte einigermassen überraschend in sie eindringen und den Verkehr vollziehen konnte. Das Opfer wurde also letztendlich - in den Worten des Gerichts - durch den Beschuldigten «übertölpelt».

\section{bb)Ausgang des Verfahrens}

21 Das Obergericht sprach den Beschuldigten vom Vorwurf der Vergewaltigung frei. Dessen Vorgehen (auch wenn er zum massgeblichen Zeitpunkt erst 16 Jahre alt gewesen sei) gegenüber einem 15-jährigen Mädchen sei zwar «egoistisch, rücksichtslos, kaltherzig und eigentlich niederträchtig» gewesen. Eine ausreichend erhebliche Einwirkung auf die Freiheit der sexuellen Selbstbestimmung im Sinne einer Nötigung gemäss Art. 190 Abs. 1 StGB liege jedoch nicht vor. Dass der Beschuldigte das Opfer be-

33 Urteil des Obergerichts Zürich SB150361 vom 8. Februar 2016. Das Gericht stützte seine rechtliche Bewertung massgeblich auf die Aussagen des Opfers und merkte diesbezüglich an: «Selbst wenn mit der Vorinstanz entgegen den Bestreitungen des Beschuldigten vollumfänglich auf die Darstellungen der Privatklägerin abgestellt wird, lässt sich daraus kein nötigendes Verhalten des Beschuldigten ableiten, welches den Tatbestand der Vergewaltigung erfüllen würde», vgl. a.a.O. S. 13. 
droht hätte, wurde ihm nicht zur Last gelegt. Ebenso wenig habe er im fraglichen Zeitpunkt (zusätzliche) Gewalt angewendet. Auch habe er sie nicht zum Widerstand unfähig gemacht; das Opfer hätte Bett, Schlafzimmer und Wohnung verlassen, schreien, oder den Beschuldigten wegweisen können. Diese ihr «zumutbare» Widersetzung durch physische Gegenwehr oder Flucht habe sie laut Gericht unterlassen. Die schamlose Ausnützung von Gefühlen, das hartnäckige Überreden sowie die letztlich zum Erfolg führende Täuschung seien zwar moralisch verwerflich, erfüllten jedoch nicht den objektiven Tatbestand von Art. 190 StGB.

\section{cc) Anmerkung}

Der Freispruch erscheint mit Blick auf das geltende Recht und die bundesgerichtliche Rechtsprechung korrekt: es fehlt objektiv an einem Nötigungsmittel. Der Beschuldigte hat das Opfer aber nicht - wie es das Gericht fälschlicherweise nennt - «getäuscht», sondern schlicht überrumpelt (er hat die täuschungsbedingte Überraschung ausgenutzt und so seinen Penis einführen können).

\section{dd)Fall Nr. 3: Sachverhalt}

Der Beschuldigte lernte das sich bei ihm als volljährig ausgebende Opfer in einem Club kennen, seine Annäherungsversuche blieben allerdings weitgehend erfolglos. Ein paar Wochen später trafen sich die beiden wieder im selben Club. Diesmal führte er die sichtlich angetrunkene junge Frau in den Darkroom des Clubs. Dort habe er sie nach Angaben des Opfers zum Konsum von Kokain aufgefordert. Als sie danach die Kabine verlassen wollte, habe er sie zunächst geküsst, anschliessend ihren Oberkörper zurechtgerückt, sie leicht nach vorne gebeugt und an den Armen und der Taille gehalten. So kam es zu verschiedenen sexuellen Handlungen, zuletzt auch zu ungeschütztem Geschlechtsverkehr. Das Opfer selber gab an, der Angeklagte habe keine eigentliche Gewalt angewendet und sie auch nicht bedroht. Sie habe ihm aber mehrmals klar gesagt, dass sie das nicht wolle und habe auch versucht, den Beschuldigten am Oberkörper «ein wenig» wegzustossen. Schliesslich habe sie das Ganze einfach über sich ergehen lassen, sie habe nicht die Kraft gehabt, sich zu wehren. Das Gericht erachtete die Aussagen des Opfers als glaubhaft und hielt im Urteil ausdrücklich fest, dass es für den Beschuldigten «zweifellos» erkennbar gewesen sei, dass er gegen den Willen des Opfers den Geschlechtsverkehr vollzog.

\section{ee) Ausgang des Verfahrens}

24 Der Beschuldigte wurde vom Vorwurf der Vergewaltigung freigesprochen. Aufgrund der «nahezu» fehlenden aktiven Abwehr habe es nur eine geringfügige Kraftanstrengung des Beschuldigten gebraucht, womit die erforderliche Intensität beim Nötigungsmittel «Gewalt» nicht erreicht sei. Auch das Tatmittel des «Bedrohens» war nicht gegeben und ebenso wenig dasjenige des «Unter-psychischenDruck-Setzens». Es habe weder eine soziale noch eine körperliche Dominanz bestanden, welche dem Opfer weitergehenden Widerstand unzumutbar gemacht hätte. Auch das «Herbeiführen von Widerstandsunfähigkeit» als letztes gesetzlich genanntes Tatmittel liege nicht vor. 
Das Gericht erachtete auch Art. 193 StGB (Ausnützen einer Notlage, ein mit maximal drei Jahren Freiheitsstrafe bedrohter und damit bloss als Vergehen ausgestalteter Straftatbestand) als nicht anwendbar. Es könne nicht von einer vorbestehenden Zwangslage ausgegangen werden, die vom Beschuldigten ausgenutzt worden sei.

\section{c) Art. $198 \mathrm{StGB}$ als Auffangtatbestand?}

26 Es ergeben sich in der Lebenswirklichkeit also Fälle, in denen ein (sexuell mündiges) Opfer ausdrücklich «Nein» sagt, die sexuelle Handlung aber dennoch duldet, ohne sich körperlich zu wehren, so dass der Täter keine Nötigungsmittel und insbesondere keine Gewalt anwenden muss. Solche Übergriffe können im schweizerischen Strafrecht - ein rechtzeitig gestellter Strafantrag vorausgesetzt - unter Umständen hilfsweise als sexuelle Belästigung nach Art. 198 StGB mit Busse bestraft werden. Dies ist etwa im folgenden Fall geschehen:

\section{aa) Fall Nr. 4: Sachverhalt}

Nach den als glaubhaft eingestuften Schilderungen des Opfers begann der Beschuldigte, sie nach einem gemeinsamen Ausgang bei sich zuhause auf dem Sofa zu streicheln und zu küssen. Nachdem sie den Kuss kurz erwiderte, gab sie anschliessend $\mathrm{zu}$ verstehen, dass sie nicht mehr wolle. Der Beschuldigte ignorierte ihre Äusserungen und öffnete ihren $\mathrm{BH}$. Sie sprang auf und sagte, sie wolle heimgehen. Er zog sie wieder auf das Sofa und nach einem kurzen Gespräch näherte er sich ihr erneut, und versuchte, ihr die Hose runterzuziehen, was ihm wegen des Gurtes nicht gelang.
Wiederum gelang es ihm, den $\mathrm{BH}$ und die Bluse zu öffnen und er fing an, ihre Brüste zu küssen. Mit dem Ziel, ihn von ihrem Busen fernzuhalten, begann sie, ihn auf den Mund zu küssen und drehte sich von ihm weg. In dieser Position führte er seine Hand in ihre Hose, worauf sie diese hinauszog. Er wiederholte dies ein- oder zweimal, wobei es ihm gelang, das Opfer direkt an der Vagina anzufassen und $\mathrm{zu}$ streicheln. Erneut sprang sie auf und forderte nochmals, dass er sie gehen lassen sollte. Daraufhin fuhr er sie nach Hause.

\section{bb)Ausgang des Verfahrens}

28 Die Staatsanwaltschaft kam zum Schluss, dass die Schilderung des Opfers glaubhaft war, dass jedoch die Tatmittel des Beschuldigten «nicht die Intensität erreicht haben, damit im Sinne von Art. 189 StGB von Bedrohung, Gewaltanwendung, Unter-psychischen-DruckSetzen oder Zum-Widerstand-Unfähigmachen gesprochen werden kann.» Anders gewendet: Das blosse (wissentliche) Übergehen einer Ablehnung (Anfassen des Opfers an der Vagina, obwohl dieses mehrfach die Hand des Beschuldigten aus ihrer Hose hinauszog) erfüllt den Tatbestand von Art. 189 StGB nicht. Das Verhalten des Beschuldigten, welches laut Staatsanwaltschaft das «zulässige Mass an Drängen» überschritten habe, wurde deshalb bloss als tätliche sexuelle Belästigung i.S.v. Art. 198 Abs. 2 StGB qualifiziert.

29 Anders als früher in Deutschland existiert in der Schweiz mit Art. 198 StGB also immerhin eine Art Auffangtatbestand für alle nicht-einverständlichen se- 
xuellen Übergriffe. 34 Zufriedenstellend ist diese Lösung allerdings nicht. Problematisch ist dabei zum einen das Strafantragserfordernis, da es nicht allen Opfern gelingt, sich innert drei Monaten zu einer Anzeige durchzuringen.35 Bedenklich ist zum anderen die damit verbundene Qualifikation des Übergriffs als Übertretung und damit als blosse Bagatelle, die lediglich mit Busse bestraft werden kann. Diese Einstufung und die Bezeichnung eines Vorfalls als «Belästigung» mag bei weniger eingriffsintensiven Zudringlichkeiten wie einem flüchtigen Anfassen des Busens über den Kleidern gerechtfertigt sein, nicht jedoch, wenn wie im obigen Fall eine Person gegen ihren Willen an den nackten Geschlechtsteilen angefasst wird oder - wie in anderen Fällen - gar penetriert wird. ${ }^{6}$

34

In Urteil des Bundesgerichts 6B_1076/2015 vom 13. April 2016 wurde in einem Fall eines offenkundig nicht-einverständlichen Sexualkontakts eine Strafbarkeit nach Art. 193 «konstruiert». Diese «Lösung» überzeugt jedoch nicht: Erstens lässt sich längst nicht in jedem Fall ein Abhängigkeitsverhältnis oder eine Notlage bejahen. $\mathrm{Zu}-$ dem soll Art. 193 StGB typischerweise Fälle von sexuellen Übergriffen erfassen, in denen das Opfer «widerwillig» eine Einwilligung erteilt, vgl. dazu auch Urteil des Bundesgerichts 6B_69/2018 vom 11. Juni 2018 E. 5.1.

35 Eingehend dazu Reinhard Plassmann, Weshalb Opfer sexueller Gewalt manchmal erst spät Anzeige erstatten, in: Gysi/Rüegger (Hrsg.), Handbuch sexualisierte Gewalt. Therapie, Prävention und Strafverfolgung, Bern 2018, S. 245 ff.

36 Eingehend Scheidegger (Fn. 3), Rz. 604 ff., 612. Kritisch zur Erfassung des sog. «Stealthings» als blosse Übertretung auch Mohamad El-Ghazi, Die strafrechtliche Bewertung des sogenannten Stealthings, SJZ 115/2019, S. 675 ff., 682 . In Österreich, dessen Sexualstrafrecht bis 2016 ähnlich ausgestaltet war wie das schweizerische, wurde die Einführung des neuen Tatbestandes $\S 205 a$ AStGB «Verletzung der sexuellen Selbstbestimmung» mit ebendiesen Argumenten begründet, vgl. Erläuterungen zum Ministerialentwurf des österreichischen Strafrechtsänderungsgesetzes 2015, 98/ME XXV.GP, S. 26.

\section{Kein Widerstand, kein Vorsatz, kein Sexualdelikt?}

3o Eine zweite Fallgruppe umfasst Konstellationen, in denen der fehlende Widerstand zusätzlich auf u.E. problematische Weise mit Fragen des Vorsatzes verknüpft wird. So wird einem Beschuldigten aufgrund der fehlenden, dem Opfer aber vermeintlich zumutbaren Gegenwehr bisweilen zugestanden, dass er den entgegenstehenden Willen gar nicht erkannt habe. So verhielt es sich etwa im Bundesgerichtsurteil 6B_311/2011 vom 19. Juli 2011. Die Frau hatte den Beschuldigten mehrmals gebeten, sie in Ruhe zu lassen, sich aber anschliessend nicht weiter zur Wehr gesetzt, sondern sich während des Geschlechtsverkehrs still und reglos verhalten. Das Bundesgericht schützte in diesem Urteil die Ansicht der Vorinstanz, «qu'au vu du comportement de la recourante, l'intimé avait $\mathrm{pu}$ croire qu'elle acceptait finalement d'entretenir une relation sexuelle furtive avec lui.»37 Ähnlich verhielt es sich auch in einigen der uns zugesandten Fälle, in denen das Opfer zwar seine Ablehnung manifestierte, der Beschuldigte aber kein Nötigungsmittel anwenden musste und in der Folge nicht nur der objektive, sondern auch - quasi vorsorglich - der subjektive Tatbestand verneint wurde. Zur Veranschaulichung der folgende Fall:

\section{aa) Fall Nr. $5^{38}$ :Sachverhalt}

31 Das Opfer hatte den Beschuldigten während eines Aufenthalts in einer Klinik kennengelernt, wo sie sich anfreundeten.

37 Siehe Urteil des Bundesgerichts 6B_311/2011 vom 19. Juli 2011 E. 4.4.2

38 Schilderung und Begründung gemäss Geschädigtenvertretung, da gegen das freisprechende Urteil keine Berufung angemeldet wurde und keine schriftliche Urteilsbegründung vorliegt. 
Er besuchte sie dann einmal bei ihr zu Hause. Zuerst seien sie auf dem Balkon gesessen und hätten etwas Alkohol getrunken. Dort habe der Beschuldigte versucht, die Hand des Opfers zu halten. Sie habe ihm diese mehrfach entzogen. Danach sei das Opfer reingegangen und habe sich auf das Bett gelegt, weil sie sich nicht gut gefühlt habe und der Beschuldigte habe sich neben sie gelegt. Nach den Aussagen des Opfers habe sie auf der Seite gelegen und der Beschuldigte habe sie auf den Bauch gedreht, ihr die Tops hochgeschoben und angefangen, sie zu massieren. Sie habe zu ihm gesagt, er solle aufhören. Er habe jedoch nichts erwidert und ungestört weitergemacht. Danach habe der Beschuldigte ihr die Hosen und Unterhosen ausgezogen. Der Beschuldigte führte dazu aus, er habe nicht gewusst, ob sie damit einverstanden gewesen sei. Sie habe nicht klar gesagt, er solle aufhören. Für ihn sei es ein «nein, aber...» gewesen. Anschliessend habe er sie oral stimuliert. Danach sei er hochgerutscht, so dass sein Glied in der Nähe des Intimbereichs des Opfers zu liegen kam. Sie habe daraufhin versucht, ihn mit ganzer Kraft wegzustossen, woraufhin er gefragt habe, was los sei. Er habe den Geschlechtsverkehr nicht vollzogen, jedoch wiederum begonnen, sie im Intimbereich zu berühren, woraufhin sie ins Badezimmer geflüchtet sei. Von dort aus schrieb sie ihm eine Textnachricht, er solle nach Hause gehen.

\section{bb)Ausgang des Verfahrens}

Nachdem die Staatsanwaltschaft das Verfahren zunächst einstellen wollte, wurde nach einer erfolgreichen Beschwerde doch noch Anklage wegen sexueller Nötigung, Vergewaltigung, evtl. sexueller Belästigung erhoben. Das Gericht sprach den Beschuldigten letztendlich aber frei, da es der Auffassung war, dass objektiv bereits das erforderliche Nötigungsmittel fehle bzw. sich der Täter nicht über einen genügend geäusserten Willen des Opfers hinwegsetzt habe. Das Gericht erachtete es zudem als unklar, ob der Beschuldigte tatsächlich wusste oder zumindest in Kauf nahm, dass sich das Opfer durch seine Berührungen belästigt fühlte. An letzterem scheiterte gemäss Angaben der Opfervertreterin auch eine Strafbarkeit wegen sexueller Belästigung.

33 Die anzutreffende Praxis, dass auch in Konstellationen mit einer ausdrücklichen verbalen Willensäusserung des Opfers dem Beschuldigten zugestanden wird, er habe den entgegenstehenden Willen nicht erkannt bzw. nicht erkennen können, ist problematisch. Zwar ist unbestritten, dass die irrtümliche Annahme des Beschuldigten, das Opfer sei mit den sexuellen Handlungen tatsächlich einverstanden gewesen, ein Sachverhaltsirrtum ist und folglich den Vorsatz des Täters entfallen lässt.39 Richtig ist auch, dass wer durch die (bewusste) Anwendung eines Nötigungsmittels den Willen des Opfers beugt und dieses dadurch zur Duldung einer sexuellen Handlung veranlasst, sich i.d.R. nicht auf einen Irrtum und damit auf fehlenden Vorsatz berufen

$39 \overline{\text { Vgl. Art. } 13 \text { StGB. Mangels Fahrlässigkeitsstraf- }}$ barkeit bleibt ein solcher Täter straflos, vgl. statt vieler Günter Stratenwerth/Guido Jenny/Felix Bommer, Schweizerisches Strafrecht, Besonderer Teil 1: Straftaten gegen Individualinteressen, 7. Aufl., Bern 2010, § 8 Rz. 15. Vgl. Kritik und Lösungsansätze etwa bei Tatjana Hörnle, Der Irrtum über das Einverständnis des Opfers bei einer sexuellen Nötigung, in: ZStW 112, 2000, S. 356 ff.; Günter Jerouschek, Der irrtumsgeneigte Vergewaltigungstäter. Überlegungen zum Verhältnis von $\S 177$ und $\S 223$ StGB und zur Auffangfunktion von $\S 230$ StGB im Strafprozess, in: JZ 1992, S. 227 ff. 
kann, wenn das Opfer angesichts des Zwangs kooperiert.40 Denn hätte der Beschuldigte tatsächlich angenommen, das Opfer sei einverstanden, wozu dann die Nötigung?41 In manchen Entscheiden wird nun aber augenscheinlich der problematische Umkehrschluss gezogen, dass ohne die Anwendung eines Nötigungsmittels - trotz entgegenstehender Willensäusserung - der ablehnende Wille des Opfers für den Beschuldigten nicht erkennbar gewesen sei. Dieser Umkehrschluss ist u.E. falsch: Nur weil der Beschuldigte kein Nötigungsmittel wie Gewalt oder Bedrohung anwenden muss, bedeutet dies nicht, dass er deshalb die fehlende Einwilligung nicht erkennen kann bzw. erkannt hat. Sagt das Opfer explizit «Nein» oder ergibt sich die fehlende Einwilligung sonst wie klar aus den Umständen, ist das Vorbringen, der Beschuldigte sei doch irgendwie von einem Einverständnis ausgegangen, wenig plausibel.42 Vielmehr ist davon auszugehen, dass dann das Übergehen des entgegenstehenden Willens zumindest in Kauf genommen wurde. 43

Der Umkehrschluss hat zudem eine bedeutsame Konsequenz: Denn gesteht man einem Beschuldigten, der kein Nötigungsmittel anwenden musste, zu, er habe trotz eines verbalen «Neins» den entgegenstehenden Willen des Opfers nicht erkannt, so muss ihm dieser Irrtum zwangsläufig auch in Bezug auf den «Auffangtatbestand» von Art. 198 StGB

$40 \overline{\text { Vgl. Scheidegger (Fn. 3), Rz. 58, unter Hinweis }}$ auf Urteil des Bundesgerichts 6B_278/2011 vom 16. Juni 2011 E. 3.3.3.

41 Vgl. Hörnle (Fn. 39), S. 357.

42 So auch Tatjana Hörnle: in Leipziger Kommentar, Strafgesetzbuch, Bd. 6, 12. A., Berlin 2009, $\S 177$ Rz. 127.

43 Vgl. dazu auch Urteil des Bundesgerichts 6B_267/2016 vom 15. Februar 2017 E. 5.2. zugutegehalten werden. Und damit bleibt das Verhalten im Ergebnis gänzlich straflos.

\section{Kritische Diskussion und kriminalpolitische Einbettung}

\section{Materielles Recht als Hürde}

35 Mit den Fallbeispielen ist aufgezeigt worden, dass eine strafrechtliche Verfolgung von nicht-einverständlichen sexuellen Handlungen mitunter auch am materiellen Recht scheitert. Das blosse Handeln gegen den Willen bzw. ohne Einwilligung wird von Art. 189 bzw. 190 StGB nicht erfasst. Der Auffangtatbestand der tätlichen sexuellen Belästigung (Art. 198 Abs. 2 StGB) greift erstens aufgrund des Strafantragserfordernisses nicht immer und erlaubt zweitens keine adäquate Sanktionierung des begangenen Unrechts. So berichteten denn auch mehrere Anwältinnen und Opferhilfeberaterinnen, dass Opfer, die einen solchen eingriffsintensiven «Übergriff ohne Nötigung» erlebt haben, dieses «Ausweichen» auf Art. 198 StGB nicht nachvollziehen können, die Einstufung ihres Übergriffs als blosse sexuelle Belästigung mitunter sogar als Hohn empfinden. Dieses Bedürfnis nach «fair labeling», d.h. das Bedürfnis, dass «widely felt distinctions between kinds of offences and degrees of wrongdoing are respected and signaled by the law, and that offences should be divided and labeled so as to represent fairly the nature and magnitude of the law-breaking»44, ist nicht überraschend. Es spielt sowohl für Täter als auch für die

$44 \overline{\text { Andrew Ashworth/Jeremy Horder, Principles of }}$ Criminal Law, 7. Aufl., Oxford 2013, S. 77; dieser Auffassung folgend auch Stuart Green, Consent and the Grammar of Theft Law, in: Cardozo Law Review, 6/2007, S. 2505 ff., 2516 f. 
Opfer und auch für die gesamte Rechtsgemeinschaft eben nicht nur eine Rolle, ob oder dass ein Täter überhaupt verurteilt wird, sondern auch, gestützt auf welchen Tatbestand er verurteilt wird. 45 Denn bereits die Bezeichnung der Tat und ihre systematische Einordnung im Gefüge des Strafgesetzbuches gibt Aufschluss darüber, wie das begangene Unrecht gesellschaftlich bewertet wird. Der Aspekt des «fair labeling» spielt auch bei der Frage der geschlechterneutralen Definition des Sexualstrafrechts eine wichtige Rolle - dies als blosse Kosmetik46 abzutun, erscheint nicht sachgerecht. 47 Hierauf wird später noch zurückzukommen sein.

Durch die Konzipierung des Vergewaltigungstatbestandes als Nötigungsdelikt wird deutlich, dass der Gesetzgeber vom Opfer grundsätzlich erwartet, dass es den ungewollten sexuellen Handlungen Widerstand entgegensetzt, den der Täter mit den gesetzlich genannten Nötigungsmitteln brechen muss. Damit wird die Verantwortung dafür, ob ein sexueller Übergriff als erhebliches Unrecht gewertet werden kann, zumindest partiell dem Opfer übertragen. Fehlt dieser Widerstand, kommt eine Strafbarkeit wegen Art. 189 bzw. 190 StGB nur infrage, wenn Umstände vorliegen, die dessen Fehlen «nachvollziehbar» erklären - so etwa, wenn sich das Opfer in einer ausweglo-

$45 \overline{\text { Vgl. zum Ganzen James Chalmers/Fiona Lever- }}$ ick, Fair labelling in criminal law, in: Modern Law Review, 2/2008, S. 217 ff., 226.

46 So aber Marcel A. Niggli/Stefan Maeder, Beischlaf, parlamentarische Vorstösse und andere erregende Dinge, in: AJP 2016/9, S. 1159 ff., 117; ähnlich im Zusammenhang mit der hier geforderten Reform auch Ständerat Jositsch in der Sendung «club» vom 28. Januar 2020, Sexualstrafrecht - Wenn ein «Nein» nicht reicht.

47 Eingehend dazu Scheidegger (Fn. 3), Rz. 655 ff. sen Lage befand oder in einem eigentlichen «Klima der Gewalt» mit dem Täter zusammenlebt o.ä. $4^{8}$ Diese nachträgliche, von Strafverfolgung und Gerichten vorgenommene Bewertung, ob und inwieweit einem Opfer ein über eine eindeutige Willensäusserung hinausgehender Widerstand zumutbar gewesen wäre, ist problematisch. Denn damit wird verkannt, dass für viele Opfer ein solcher Widerstand in der Realität schlicht nicht möglich ist 49 - auch wenn dies für das urteilende Gericht hinterher nicht nachvollziehbar oder nicht plausibel erscheinen mag. ${ }^{50}$ Hierbei wäre das Verständnis entscheidend, dass die sog. tonische Immobilität eine normale biologische Reaktion des Opfers auf den von ihm als Bedrohung wahrgenommenen Übergriff ist. ${ }^{11}$ Wenn nun aber eine Verfahrenseinstellung oder ein Freispruch u.a. damit begründet wird, das Opfer habe den ihm «zumutbaren» Widerstand unterlassen, weil es die (vermeintlich) verfügbaren Abwehr- oder Fluchtmöglichkeiten nicht

$48 \overline{\text { Vgl. etwa Urteil des Bundesgerichts 6B_298/2008 }}$ vom 1. Juli 2008, wo eine solche «ausweglose Situation» bejaht wurde, da die «Wehrfähigkeit» des Opfers aufgrund einer starken psychischen und physischen Belastung herabgesetzt gewesen sei.

49 Eingehend dazu etwa Veronique Valliere, Understanding Victims of Interpersonal Violence: A Guide for Investigators and Prosecutors, New York 2020, S. 133 ff. mit Hinweisen; Anna Möller/Hans Peter Söndergaard/Lotti Helström, Tonic immobility during sexual assault - a common reaction predicting post-traumatic stress disorder and severe depression, in: Acta Obstetricia et Gynecologica Scandinavica, 2017, S. $932 \mathrm{ff}$.

50 Problematisch deshalb die Ausführungen von Jetzer/Gfeller (Fn. 4), wonach die Frau im vieldiskutierten Urteil des Bundesgerichts 6B_912/2009 vom 22. Februar 2010 «eben gerade nicht wie «wie gelähmt»» gewesen sei. Die Lektüre des zweitinstanzlichen Urteils (Fn. 23) zeigt nämlich, dass die Frau schockiert war und sich «wie eine Puppe» vorgekommen sei, was durchaus auf eine Schockstarre hindeuten könnte.

51 Möller/Söndergaard/Helström (Fn. 49), S. 936. 
genutzt und sich bloss verbal widersetzt habe, suggeriert dies dem Opfer, es habe eben doch eine Art «Mitschuld» am sexuellen Übergriff. Aus der Opferforschung ist bekannt, dass sich Opfer von Sexualdelikten ohnehin häufig Vorwürfe machen, sich nicht «besser» gewehrt zu haben.52 Die Bewältigung des Übergriffs wird zusätzlich erschwert, wenn ein Opfer im Urteil lesen muss, «dass es aufgrund der konkreten Verhältnisse in besagter Nacht nicht zum wiederholten Geschlechtsverkehr gekommen wäre, wenn [das Opfer] den ihr zumutbaren Widerstand geleistet hätte».53

\section{Reformvorschläge}

37 Dass Änderungsbedarf besteht und insbesondere Art. 198 StGB als Auffangtatbestand unzureichend ist, wird mittlerweile auch in der Politik zunehmend anerkannt. 54 Wie die notwendigen Änderungen umgesetzt werden sollen, ist allerdings noch offen. Mehrere Varianten sind denkbar, wie Coninx und Scheidegger anhand einer Auslegeordnung aufgezeigt haben.55 Zwei grundlegende Fragen stehen dabei im Vordergrund:

Zunächst muss geklärt werden, ob ein Vetomodell («Nein heisst Nein») oder ein Zustimmungsmodell ( Nur Ja heisst Ja») Grundlage einer neuen Strafnorm sein soll. Das Vetomodell könnte etwa

$5^{2}$ Vgl. Jan Gysi, Fünf Konzepte zur Veranschaulichung komplexer Dynamiken bei sexualsierter Gewalt; in: Gysi/Rüegger (Hrsg.), Handbuch sexualisierte Gewalt. Therapie, Prävention und Strafverfolgung, Bern 2018, S. $77 \mathrm{ff}$.

53 Urteil des Kantonsgerichts Wallis P1 0866 vom 17. September 2009 E. 6.e.cc.bbb.

54 Immerhin soll der Revisionsbedarf im Sexualstrafrecht vertieft geprüft werden, vgl. Medienmitteilung der RK-S vom 17. Januar 2020.

55 Anna Coninx/Nora Scheidegger, Änderungsbedarf im Sexualstrafrecht - Auslegeordnung, 2019. mit der gesetzlichen Formulierung «Wer gegen den Willen einer anderen Person sexuelle Handlungen an dieser vornimmt (...)» umgesetzt werden. Das Zustimmungsmodell hingegen würde mit der Formulierung «Wer ohne Zustimmung einer Person einer anderen Person sexuelle Handlungen an dieser vornimmt (...)» verankert. In jenen Fällen, in denen ganz offensichtlich keine Zustimmung vorliegt (überraschende oder unerwartete sexuelle Handlungen; explizites oder konkludentes «Nein») dürfte das Kriterium der fehlenden Zustimmung selbsterklärend sein. In weniger klaren Fällen, die sich etwa im Rahmen einer (zunächst) einvernehmlichen sexuellen Begegnung abspielen, würde sich die $\mathrm{Zu}-$ stimmungslösung kaum vom Vetomodell unterscheiden. Denn in diesen Fällen wird auch die Zustimmungslösung nicht zuletzt aus Gründen des Vorsatznachweises erfordern, dass der Meinungsumschwung und damit das Fehlen einer Zustimmung durch explizites oder zumindest konkludentes «Nein» zum Ausdruck kommt. Um zu vermeiden, dass auch nach einer Reform des Sexualstrafrechts das Widerstandserfordernis gewissermassen über den subjektiven Tatbestand wieder hineingeschleust würde, indem der Täterschaft bei Fehlen einer Nötigungshandlung wie oben dargelegt regelmässig ein Irrtum zugestanden würde, wäre sicherlich eine eingehende Auseinandersetzung mit den denkbaren Irrtumskonstellationen notwendig.

39 In einem zweiten Schritt müsste entschieden werden, ob der angestrebte Paradigmenwechsel durch eine Neudefinition insbesondere von $189 \mathrm{f}$. StGB, durch Einfügung eines Grundtatbestandes bei Art. 189 f. StGB oder durch Schaffung ei- 
nes neuen, separaten Tatbestandes verwirklicht werden soll. Die letzte Variante scheint deshalb attraktiv, weil damit die Abstufung zur Vergewaltigung, wie sie heute definiert ist, klar beibehalten würde. Die neuerdings vorgeschlagene Lösung, anstatt eines neuen Tatbestands lediglich den Art. 198 StGB um einen «schweren Fall» zu ergänzen und so einen neuen Auffangtatbestand zu schaffen, 56 überzeugt u.E. nicht. Art. 198 StGB ist auf verbale und bagatellhafte körperliche Zudringlichkeiten wie etwa ein flüchtiges «Grapschen» zugeschnitten, erheblichere sexuelle Handlungen sollten daher in den vorangehenden Tatbeständen geregelt sein. Würden auch Beischlaf oder sonstige Penetrationen gegen den Willen einer Person bei der sexuellen Belästigung angesiedelt, so überzeugt dies systematisch nicht und führt dazu, dass solche Übergriffe den Anstrich eines blossen Bagatellunrechts behalten, was nicht angeht. 57

\section{Falsche Versprechungen?}

40 Die hier vorgeschlagene Reform des Sexualstrafrechts stiess vereinzelt auf Kritik. StrafverteidigerInnen und PolitikerInnen wähnten nicht weniger als die Grundprinzipien des Strafrechts und des Rechtsstaates in Gefahr. ${ }^{8}$ Auf sämtliche Punkte der Reformgegner detailliert einzugehen, würde den vorliegenden Rah-

$5 6 \longdiv { \text { So augenscheinlich das Ansinnen von Ständerat } }$ Jositsch (Fn. 46).

57 Kritisch zu dieser Lösung auch bereits Coninx/Scheidegger (Fn. 55), S. 7.

$58 \mathrm{Vgl}$. etwa Gastbeitrag StrafverteidigerInnen (Fn. 4); Statement von Ständerat Jositsch im Beitrag von Daniel Gerny, Soll Sex ohne Zustimmung eine Vergewaltigung sein? Juristen streiten über eine weitgehende Revision des Schweizer Strafrechts, in: NZZ vom 6. Januar 2020: «Hier werden strafrechtliche Prinzipien über den Haufen geworfen.» men sprengen. Nur so viel sei gesagt: Weder wäre mit einer allfälligen Reform eine Beweislastumkehr verbunden, noch würde die Unschuldsvermutung ausgehebelt.59 Gehaltvoller ist aber auf den ersten Blick der folgende Einwand: Gerade weil keine Beweislastumkehr stattfindet und auch die Unschuldsvermutung unangetastet bleibt, würde eine Reform nicht zu mehr Verurteilungen führen. Man wecke deshalb «falsche Erwartungen».60

41 Natürlich darf nicht die Vorstellung heraufbeschworen werden, dass nach einer Reform schon die blosse Behauptung, «Nein» gesagt zu haben, in jedem Fall zu einer strafrechtlichen Verurteilung führen wird. Angesichts der beweisrechtlichen Verfahrenswirklichkeit ist damit zu rechnen, dass auch künftig bei einander widersprechenden Aussagen oftmals ein Freispruch ergehen muss, wenn keine Beweise für die Anwendung eines Nötigungsmittel vorliegen. Dies dürfte auch den meisten Opfern bewusst sein. Nach Erfahrung von Opferhilfeberaterinnen erstatten aber nicht wenige Frauen Anzeige als wichtigen Schritt in ihrem Verarbeitungsprozess, obwohl sie sich aufgrund der Beweisprobleme wenig Hoffnung auf eine Verurteilung «ihres» Täters machen. ${ }^{61}$ Für diese Opfer macht es aber einen wesentlichen Unterschied, ob ein Verfahren aufgrund mangelnder Be-

$59 \overline{\text { Vgl. dazu die klaren Ausführungen des obersten }}$ schwedischen Gerichts zum neuen Sexualstrafrecht im Urteil Nr. B 1200-19 vom 11. Juli 2019, Rz. 16.

60 So Jetzer/Gfeller (Fn. 4).

61 Vgl. das Statement eines Opfers in der Sendung Input vom 18. Oktober 2018: "Ich weiss, dass es kein gerechtes Ende geben wird. Ich werde nicht gewinnen. Das ist so gut wie nie der Fall. Ich muss es tun, weil es mir das Gefühl gibt, alles getan zu haben, was in meiner Macht steht (...).» 
weisbarkeit eingestellt wird oder ob die Einstellung mit dem Hinweis erfolgt, das Verhalten des Beschuldigten stelle ohnehin kein erhebliches Unrecht dar.

42 Hinweise aus der Dunkelfeldforschung deuten darauf hin, dass sexuelle Übergriffe - verstanden als nichteinverständliche sexuelle Handlungen häufig vorkommen, ein Grossteil dieser Fälle jedoch nie angezeigt wird.62 Die geringe Anzeigebereitschaft der Opfer liegt sicherlich einerseits darin begründet, dass Opfer aus (falscher) Scham und/oder aus Furcht vor den Belastungen eines Strafverfahrens auf eine Anzeige verzichten. ${ }^{63}$ Allerdings berichten Opferberaterinnen, dass mitunter auch die

$62 \overline{\text { Vgl. gfs.bern, Befragung sexuelle Gewalt an Frau- }}$ en im Auftrag von Amnesty International Schweiz, vom 17. Mai 2019, wonach $12 \%$ der befragten Frauen seit ihrem 15. Lebensjahr Geschlechtsverkehr gegen den Willen erlebt haben. Eine EU-weite Untersuchung ermittelte ebenfalls hohe Prävalenzraten, vgl. Agentur der Europäischen Union für Grundrechte, Gewalt gegen Frauen: eine EU-weite Erhebung - Ergebnisse auf einen Blick, 2014, S. 9: «Jede zehnte Frau hat seit ihrem 15. Lebensjahr irgendeine Form der sexuellen Gewalt erfahren und jede zwanzigste Frau ist, seit sie 15 war, vergewaltigt worden.» Gemäss der gfs-Befragung, S. 16, haben nur 8\% der Befragten, die sexuelle Gewalt erfahren haben, Anzeige erstattet. In einer Befragung des Bundesministeriums für Familie, Senioren, Frauen und $\mathrm{Ju}$ gend zur Lebenssituation, Sicherheit und Gesundheit von Frauen in Deutschland (2005), S. 208, zeigte sich ein ähnliches Bild. Eingehend zum Forschungstand Angelika Treibel/Dieter Dölling/Dieter Hermann, Das Anzeigeverhalten Betroffener sexueller Übergriffe, in: Wazlawik et al. (Hrsg), Sexuelle Gewalt in pädagogischen Kontexten - Aktuelle Forschungen und Reflexionen, Wiesbaden 2019, S. 125 ff. Eingehend auch Klaus Laubenthal, Kriminologische Aspekte zu Vergewaltigung und sexueller Nötigung - unter besonderer Berücksichtigung des Auseinanderfallens von Strafanzeigen und Verurteilungen. Stellungnahme im Abschlussbericht der Reformkommission zum Sexualstrafrecht vom 19. Juli 2017, S. $1128 \mathrm{ff}$.

63 Vgl. eingehend Treibel/Dölling/Hermann (Fn. 62), S. 128 ff.; Befragung gfs.bern (Fn. 62), S. 16 f. aktuelle Rechtslage dazu beitrage, dass Opfer den erlebten sexuellen Übergriff gar nicht erst anzeigen. Manche Opfer würden die juristische Beratung als ernüchternd erleben, da ihnen dargelegt werde, dass die Gewalt, die sie selber instinktiv als massive Verletzung ihrer sexuellen Integrität einordnen, juristisch wegen der fehlenden Nötigungshandlung wohl nicht als solche eingestuft würde. ${ }^{64}$ ExpertInnen von Opferhilfeberatungsstellen sind deshalb überzeugt, dass eine Reform des Sexualstrafrechts Opfer in ihrer instinktiven Wahrnehmung bestärken und letztlich dazu führen würde, dass diese vermehrt Anzeige erstatten. ${ }^{65}$

43 Ganz zuletzt darf nicht vergessen werden, dass es in einzelnen Fällen durchaus möglich wäre, die fehlende Einvernehmlichkeit des Sexualkontakts nachzuweisen - etwa in Fällen, in denen der Beschuldigte geständig ist oder der Vorfall etwa mittels Kamera dokumentiert wurde. ${ }^{66}$ Erste Erfahrungen mit den neuen Regelungen etwa in Deutschland oder Schweden zeigen denn auch, dass es

$64 \overline{\text { Vgl. auch Christian Diesen/Eva Diesen, Sex crime }}$ legislation: Proactive and anti-therapeutic effects, in: International Journal of Law and Psychiatry, 2010, S. 329 ff., 331, wonach sich eine Rechtslage wie die schweizerische dahingehend auswirken könne, dass sie Opfern Anlass gebe, «to distrust society [and] the legal system.» Vgl. auch Sarah Seifarth/Heike Ludwig, Dunkelfeld und Anzeigeverhalten bei Delikten gegen die sexuelle Selbstbestimmung. Ergebnisse einer Untersuchung zur Erforschung von Anzeigemotivation und Anzeigeverhalten bei sexueller Nötigung und Vergewaltigung, Monatsschrift für Kriminologie und Strafrechtsreform 5/2016, S. 237 ff., wonach durch «Reformierungen» des Strafgesetzbuches grds. das Vertrauen in das Rechtssystem gestärkt und damit die Anzeigebereitschaft erhöht werden könnte.

65 Medienmitteilung «Nationales Fachgremium Sexuelle Gewalt an Frauen» (Fn. 3).

66 Vgl. beispielsweise das Urteil des Kantonsgerichts St. Gallen ST.2016.105 vom 22. August 2017. 
durchaus zu Anklagen und Verurteilungen kommt - wenn auch in überschaubarer Anzahl. ${ }^{67}$ Die Notwendigkeit oder Legitimation einer Strafnorm wird aber bekanntlich nicht an der Anzahl der Verurteilungen gemessen.

67 Die Berliner Oberstaatsanwältin Ines Karl spricht von «wenigen Urteilen», vgl. Lisa-Marie Leuteritz, Was der «Nein heißt Nein»-Paragraph verbessert hat - und was nicht, in: Hannoversche Allgemeine vom 25. November 2018; in Schweden wurden für das Programm I lagens namn [«Im Namen des Gesetzes»] 6o Verurteilungen analysiert, die seit der Reform ergangen sind, wobei sich zeigte, dass das neue Gesetz in sieben Fällen entscheidend war, wobei einer der Fälle eine sog. «unachtsame» Vergewaltigung betraf. 